\title{
Hochschild homology, Frobenius homomorphism and Mac Lane homology
}

\author{
TEIMURAZ PIRASHVILI
}

\begin{abstract}
We prove that $\mathrm{H}_{i}(A, \Phi(A))=0, i>0$. Here $A$ is a commutative algebra over the prime field $\mathbb{F}_{p}$ of characteristic $p>0$ and $\Phi(A)$ is $A$ considered as a bimodule, where the left multiplication is the usual one, while the right multiplication is given via Frobenius endomorphism and $\mathrm{H}_{\bullet}$ denotes the Hochschild homology over $\mathbb{F}_{p}$. This result has implications in Mac Lane homology theory. Among other results, we prove that HML. $(A, T)=0$, provided $A$ is an algebra over a field $K$ of characteristic $p>0$ and $T$ is a strict homogeneous polynomial functor of degree $d$ with $1<d<\operatorname{Card}(K)$.
\end{abstract}

55P43, 16E40; 19D55, 55U10

\section{Introduction}

In this short note we study Hochschild and Mac Lane homology of commutative algebras over the prime field $\mathbb{F}_{p}$ of characteristic $p>0$. Let us recall that Mac Lane homology is isomorphic to the topological Hochschild homology (Pirashvili-Waldhausen [13]) and to the stable $K$-theory as well (Franjou et al [4]).

Let $A$ be a commutative algebra over the prime field $\mathbb{F}_{p}$ of characteristic $p>0$ and let $\Phi(A)$ be an $A-A$-bimodule, which is $A$ as a left $A$-module, while the right multiplication is given via Frobenius endomorphism. We prove that the Hochschild homology vanishes $\mathrm{H}_{i}(A, \Phi(A))=0, i>0$. The proof makes use a simple result on homotopy groups of simplicial rings, which says that if $R_{\bullet}$ is a simplicial ring such that all rings involved in $R_{\bullet}$ satisfy $x^{m}=x, m \geq 2$ identity then $\pi_{i}\left(R_{\bullet}\right)=0$ for all $i>0$. These results has implications in Mac Lane homology theory. We extend the computation of Franjou-Lannes and Schwartz [6] of Mac Lane (co)homology of finite fields with coefficients in symmetric $S^{d}$ and divided $\Gamma^{d}$ powers to arbitrary commutative $\mathbb{F}_{p}$-algebras, provided that $d>1$. As a consequence of our computations we show that $\operatorname{HML}_{\bullet}(A, T)=0$, provided $T$ is a strict homogeneous polynomial functor of degree $d>1$ and $A$ is an algebra over a field $K$ of characteristic $p>0$ with $\operatorname{Card}(K)>d$. 
Many thanks to referee for his valuable comments and to Petter Andreas Bergh for his remarks. The author was partially supported by the grant from MEC, MTM200615338-C02-02 (European FEDER support included).

\section{When it is too easy to compute homotopy groups}

It is well known that the homotopy groups of a simplicial abelian group $\left(A_{\bullet}, \partial_{\bullet}, s_{\bullet}\right)$ can be computed as the homology of the normalized chain complex $\left(N_{\bullet}\left(A_{\bullet}\right), d\right)$, where

$$
N_{n}\left(A_{\bullet}\right)=\left\{x \in A_{n} \mid \partial_{i}(x)=0, i>0\right\}
$$

and the boundary map $N_{n}\left(A_{\bullet}\right) \rightarrow N_{n-1}\left(A_{\bullet}\right)$ is induced by $\partial_{0}$. Our first result shows that if $A \bullet$ has a simplicial ring structure and the rings involved in $A \bullet$ satisfy extra conditions then homotopy groups are zero in positive dimensions. This fact is an easy consequence of the following result which is probably well known.

Lemma 1 Let $R_{\bullet}$ be a simplicial object in the category of not necessarily associative rings and let $x, y \in N_{n}\left(R_{\bullet}\right)$ be two elements. Assume $n>0$ and $x$ is a cycle. Then the cycle $x y \in N_{n}\left(R_{\bullet}\right)$ is a boundary.

Proof Consider the element

$$
z=s_{0}(x y)-s_{1}(x) s_{0}(y) .
$$

Then we have

$$
\partial_{0}(z)=x y-\left(s_{0} \partial_{0}(x)\right) y=x y .
$$

Moreover,

$$
\partial_{1}(z)=x y-x y=0 .
$$

We also have

$$
\partial_{2}(z)=\left(s_{0} \partial_{1}(x)\right)\left(s_{0} \partial_{1}(y)\right)-x\left(s_{0} \partial_{1}(y)\right)=0 .
$$

Similarly for all $i>2$ we have

$$
\partial_{i}(z)=\left(s_{0} \partial_{i-1}(x)\right)\left(s_{0} \partial_{i-1}(y)\right)-\left(s_{1} \partial_{i-1}(x)\right)\left(s_{0} \partial_{i-1}(y)\right)=0 .
$$

Hence $z$ is an element of $N_{n+1}\left(R_{\bullet}\right)$ with $\partial(z)=x y$.

Corollary 2 Let $R_{\bullet}$ be a simplicial ring. If the rings involved in $R_{\bullet}$ satisfy $x^{m}=x$ identity for $m \geq 2$, then

$$
\pi_{n}\left(R_{\bullet}\right)=0, \quad n>0
$$

Algebraic $8 \mathcal{G}$ Geometric Topology, Volume 7 (2007) 
Proof Take a cycle $x \in N_{n}\left(R_{\bullet}\right), n>0$. Then the class of $x=x x^{m-1}$ in $\pi_{n}\left(R_{\bullet}\right)$ is zero.

Remark A more general fact is true. Let $\mathbf{T}$ be a pointed algebraic theory (Schwede [15]) and let $X_{\bullet}$ be a simplicial object in the category of $\mathbf{T}$-models [15]. Then $\pi_{1}\left(X_{\bullet}\right)$ is a group object in the category of $\mathbf{T}$-models, while $\pi_{i}\left(X_{\bullet}\right)$ are abelian group objects in the category of $\mathbf{T}$-models for all $i>1$. Thus $\pi_{i}\left(X_{\bullet}\right)=0, i \geq 1$ provided all group objects are trivial. This is what happens for the category of rings satisfying the identity $x^{m}=x, m \geq 2$. Another interesting case is the category of Heyting algebras (Esakia [3]).

\section{Hochschild homology with twisted coefficients}

In what follows the ground field is the prime field $\mathbb{F}_{p}$ of characteristic $p>0$. All algebras are taken over $\mathbb{F}_{p}$ and they are assumed to be associative. For an algebra $R$ and an $R-R$-bimodule $B$ we let $\mathrm{H}_{\bullet}(R, B)$ and $\mathrm{H}^{\bullet}(R, B)$ be the Hochschild homology and cohomology of $R$ with coefficients in $B$. Let us recall that

$$
\mathrm{H}_{\bullet}(R, B)=\operatorname{Tor}_{\bullet}^{R \otimes R^{o p}}(R, B)
$$

and

$$
\mathrm{H}^{\bullet}(R, B)=\operatorname{Ext}_{R \otimes R^{o p}}^{\bullet}(R, B) .
$$

Moreover, let $C_{\bullet}(R, B)$ be the standard simplicial vector space computing Hochschild homology

$$
\pi_{\bullet}\left(C_{\bullet}(R, B)\right) \cong \mathrm{H}_{\bullet}(R, B) .
$$

Recall that $C_{n}(R, B)=B \otimes R^{\otimes n}$, while

$$
\begin{aligned}
\partial_{0}\left(b, r_{1}, \ldots, r_{n}\right) & =\left(b r_{1}, \ldots, r_{n}\right), \\
\partial_{i}\left(b, r_{1}, \ldots, r_{n}\right) & =\left(b, r_{1}, \ldots, r_{i} r_{i+1}, \ldots, r_{n}\right), \quad 0<i<n
\end{aligned}
$$

and

$$
\partial_{n}\left(b, r_{1}, \ldots, r_{n}\right)=\left(r_{n} b, r_{1}, \ldots, r_{n-1}\right) .
$$

Here $b \in B$ and $r_{1}, \ldots, r_{n} \in R$.

Let $n \geq 1$ be a natural number and let $A$ be a commutative $\mathbb{F}_{p}$-algebra. The Frobenius homomorphism gives rise to the functors $\Phi^{n}$ from the category of $A$-modules to the category of $A$-A-bimodules, which are defined as follows. For an $A$-module $M$ the bimodule $\Phi^{n}(M)$ coincides with $M$ as a left $A$-module, while the right $A$-module structure on $\Phi^{n}(M)$ is given by

$$
m a=a^{p^{n}} m, \quad a \in A, \quad m \in M .
$$

Algebraic $8 \mathcal{G}$ Geometric Topology, Volume 7 (2007) 
Having $A-A$-bimodule $\Phi^{n}(M)$ we can consider the Hochschild homology $\mathrm{H}_{\bullet}(A$, $\left.\Phi^{n}(M)\right)$. In this section we study these homologies. In order to state our results we need some notation. We let $\psi^{n}(A)$ be the quotient ring $A /\left(a-a^{p^{n}}\right), n \geq 1$ which is considered as an $A$-module via the quotient map $A \rightarrow \psi^{n}(A)$. Thus $\psi^{n}$ is the left adjoint of the inclusion of the category of commutative $\mathbb{F}_{p}$-algebras with identity $x^{m}=x, m=p^{n}$ to the category of all commutative $\mathbb{F}_{p}$-algebras.

Example 3 Let $n \geq 1$. If $K$ is a finite field with $q=p^{d}$ element then $\psi^{n}(K)=K$ if $n=d t, t \in \mathbb{N}$ and $\psi^{n}(K)=0$ if $n \neq d t, t \in \mathbb{N}$.

Lemma 4 Let $A$ is a commutative algebra over a field $K$ of characteristic $p>0$ with $\operatorname{Card}(K)>p^{n}$. Then $\psi^{n}(A)=0, n \geq 1$.

Proof By assumption there exists $k \in K$ such that $k^{p^{n}}-k$ is an invertible element of $K$. It follows then that the elements of the form $a^{p^{n}}-a$ generates whole $A$.

Theorem 5 Let $A$ be a commutative $\mathbb{F}_{p}$-algebra and $n \geq 1$. Then

$$
\mathrm{H}_{i}\left(A, \Phi^{n}(A)\right)=0
$$

for all $i>0$ and

$$
\mathrm{H}_{0}\left(A, \Phi^{n}(A)\right) \cong \psi^{n}(A)
$$

Proof The proof consists of three steps.

Step 1 The theorem holds if $A=\mathbb{F}_{p}[x]$ In this case we have the following projective resolution of $A$ over $A \otimes A=\mathbb{F}_{p}[x, y]$ :

$$
0 \rightarrow \mathbb{F}_{p}[x, y] \stackrel{\eta}{\rightarrow} \mathbb{F}_{p}[x, y] \stackrel{\epsilon}{\rightarrow} \mathbb{F}_{p}[x] \rightarrow 0 .
$$

Here $\epsilon(x)=\epsilon(y)=x$ and $\eta$ is induced by multiplication by $(x-y)$. Hence for any $A-A$-bimodule $B$, we have $\mathrm{H}_{i}(A, B)=0$ for $i>1$ and

$$
\mathrm{H}_{0}(A, B) \cong \operatorname{Coker}(u) \text { and } H_{1}(A, B) \cong \operatorname{ker}(u),
$$

where $u: B \rightarrow B$ is given by $u(b)=x b-b x$. If $B=\Phi^{n}\left(\mathbb{F}_{p}[x]\right)$, then $u$ : $\mathbb{F}_{p}[x] \rightarrow$ $\mathbb{F}_{p}[x]$ is the multiplication by $\left(x^{p^{n}}-x\right)$ and we obtain $\mathrm{H}_{1}\left(A, \Phi^{n}(A)\right)=0$ and $\mathrm{H}_{0}\left(A, \Phi^{n}(A)\right)=\psi^{n}(A)$

Step 2 The theorem holds if $A$ is a polynomial algebra Since Hochschild homology commutes with filtered colimits it suffices to consider the case when $A=$ $\mathbb{F}_{p}\left[x_{1}, \ldots, x_{d}\right]$. By the Künneth theorem for Hochschild homology (see Mac Lane 
$\left[10\right.$, Theorem X.7.4]) we have $\mathrm{H}_{\bullet}\left(A, \Phi^{n}(A)\right)=\mathrm{H}_{\bullet}\left(\mathbb{F}[x], \Phi^{n}(\mathbb{F}[x])\right)^{\otimes d}$ and the result follows.

Step 3 The theorem holds for arbitrary $A$ We use the same method as used in the proof by Loday [9, Theorem 3.5.8]. First we choose a simplicial commutative algebra $L_{\bullet}$ such that each $L_{n}$ is a polynomial algebra, $n \geq 0$ and $\pi_{i}\left(L_{\bullet}\right)=0$ for all $i>0$, $\pi_{0}\left(L_{\bullet}\right)=A$. Such a resolution exists thanks to (Quillen [14]). Now consider the bisimplicial vector space $C_{\bullet}\left(L_{\bullet}, \Phi^{n}\left(L_{\bullet}\right)\right)$. The $s$ th horizontal simplicial vector space is the simplicial vector space $L_{\bullet}^{\otimes s+1}$. By the Eilenberg-Zilber-Cartier and Künneth theorems it has zero homotopy groups in positive dimensions and $\pi_{0}\left(L_{\bullet}^{\otimes s+1}\right)=A^{\otimes s+1}$. On the other hand the $t$ th vertical simplicial vector space of $\left.C_{\bullet}\left(L_{\bullet}, \Phi^{n}\left(L_{\bullet}\right)\right)\right)$ is isomorphic to the Hochschild complex $\left.C_{\bullet}\left(L_{t}, \Phi^{n}\left(L_{t}\right)\right)\right)$ which has zero homology in positive dimensions by the previous step. Hence both spectral sequences corresponding to the bisimplicial vector space $C_{\bullet}\left(L_{\bullet}, \Phi^{n}\left(L_{\bullet}\right)\right)$ degenerate and we obtain the isomorphism

$$
\mathrm{H}_{\bullet}\left(A, \Phi^{n}(A)\right) \cong \pi_{\bullet}\left(\psi^{n}\left(L_{\bullet}\right)\right) .
$$

Now we can use Corollary 2 to finish the proof.

Corollary 6 Let $A$ be a commutative $\mathbb{F}_{p}$-algebra, $M$ be an $A$-module and $n \geq 1$. Then there exist functorial isomorphisms

$$
\mathrm{H}_{\bullet}\left(A, \Phi^{n}(M)\right) \cong \operatorname{Tor}_{\bullet}^{A}\left(\psi^{n}(A), M\right), \quad n \geq 0
$$

and

$$
\mathrm{H}^{\bullet}\left(A, \Phi^{n}(M)\right) \cong \operatorname{Ext}_{A}^{\bullet}\left(\psi^{n}(A), M\right), \quad n \geq 0 .
$$

In particular, if $A$ is a commutative algebra over a field $K$ of characteristic $p>0$ with $\operatorname{Card}(K)>p^{n}$, then

$$
\mathrm{H}_{\bullet}\left(A, \Phi^{n}(M)\right)=0=\mathrm{H}^{\bullet}\left(A, \Phi^{n}(M)\right) .
$$

Proof Observe that $C_{\bullet}\left(A, \Phi^{n}(A)\right)$ is a complex of left $A$-modules. By Theorem 5 it is a free resolution of $\psi^{n}(A)$ in the category of $A$-modules. Hence it suffices to note that

$$
\begin{aligned}
& C_{\bullet}\left(A, \Phi^{n}(M)\right) \cong M \otimes_{A} C_{\bullet}\left(A, \Phi^{n}(A)\right), \\
& C^{\bullet}\left(A, \Phi^{n}(M)\right) \cong \operatorname{hom}_{A}\left(C_{\bullet}\left(A, \Phi^{n}(A)\right), M\right),
\end{aligned}
$$

where $C^{*}$ denotes the standard complex for Hochschild cohomology. The last assertion follows from Lemma 4. 
Example 7 It follows for instance that $\mathrm{H}^{i}\left(A, \Phi^{n}(M)\right)=0, i>0$, provided $M$ is an injective $A$-module and $n \geq 1$. In particular $\mathrm{H}^{i}\left(A, \Phi^{n}(A)\right)=0$ if $A$ is a selfinjective algebra. On the other hand if $A=\mathbb{F}_{p}\left[x_{1}, \ldots, x_{d}\right]$ then $\mathrm{H}^{i}\left(A, \Phi^{n}(A)\right)=0, i \neq d$, $n \geq 1$ and $\mathrm{H}^{d}\left(A, \Phi^{n}(A)\right)=\psi^{n}(A), n \geq 1$.

\section{Application to Mac Lane cohomology}

We recall the definition of Mac Lane (co)homology. For an associative ring $R$ we let $\mathbf{F}(R)$ be the category of finitely generated free left $R$-modules. Moreover, we let $\mathfrak{\Im}(R)$ be the category of all covariant functors from the category $\mathbf{F}(R)$ to the category of all $R$-modules. The category $\mathfrak{\Im}(R)$ is an abelian category with enough projective and injective objects. By definition (Jibladze-Pirashvili [8]) the Mac Lane cohomology of $R$ with coefficient in a functor $T \in \mathfrak{F}(R)$ is given by

$$
\mathrm{HML}^{\bullet}(R, T):=\operatorname{Ext}_{\check{\mho}(R)}^{\bullet}(I, T),
$$

where $I \in \mathfrak{F}(R)$ is the inclusion of the category $\mathbf{F}(R)$ into the category of all left $R-$ modules. One defines Mac Lane homology in a dual manner (see Pirashvili-Waldhausen [13, Proposition 3.1]). For an $R-R$-bimodule $B$, one considers the functor $B \otimes_{R}(-)$ as an object of the category $\mathfrak{F}(R)$. For simplicity we write $\operatorname{HML}_{\bullet}(R, B)$ instead of $\mathrm{HML}_{\bullet}\left(R, B \otimes_{R}(-)\right)$. There is a binatural transformation

$$
\mathrm{HML}_{\bullet}(R, B) \rightarrow \mathrm{H}_{\bullet}(R, B)
$$

which is an isomorphism in dimensions 0 and 1.

In the rest of this section we consider Mac Lane (co)homology of commutative $\mathbb{F}_{p}-$ algebras.

Lemma 8 For any commutative $\mathbb{F}_{p}$-algebra $A$ one has an isomorphism

$$
\mathrm{HML}_{2 i}\left(A, \Phi^{n}(A)\right)=\psi^{n}(A), \quad i \geq 0, n \geq 1,
$$

and

$$
\mathrm{HML}_{2 i+1}\left(A, \Phi^{n}(A)\right)=0, \quad i \geq 0, n \geq 1 .
$$

Proof According to (Pirashvili [12, Proposition 4.1]) there exists a functorial spectral sequence

$$
E_{p q}^{2}=\mathrm{H}_{p}\left(A, \mathrm{HML}_{q}\left(\mathbb{F}_{p}, B\right)\right) \Longrightarrow \mathrm{HML}_{p+q}(A, B) .
$$

Here $B$ is an $A-A$-bimodule. By the well-known computation of Breen [2], Bökstedt [1] (see also Franjou-Lannes-Schwartz [6]) we have

$$
\mathrm{HML}_{2 i}\left(\mathbb{F}_{p}, B\right)=B
$$


and

$$
\mathrm{HML}_{2 i+1}\left(\mathbb{F}_{p}, B\right)=0 .
$$

Now we put $B=\psi^{n}(A)$ and use Theorem 5 to get $E_{p q}^{2}=0$ for all $p>0$. Hence the spectral sequence degenerates and the result follows.

We now consider Mac Lane cohomology with coefficients in strict polynomial functors (Friedlander-Suslin [7]). Let us recall that the strict homogeneous polynomial functors of degree $d$ form an abelian category $\mathfrak{P}_{d}(A)$ and there exist an exact functor $i: \mathfrak{P}_{d}(A) \rightarrow \mathfrak{F}(A)$ (Franjou et al [5]). For an object $T \in \mathfrak{P}_{d}(A)$ we write HML. $(A, T)$ instead of HML $(A, i(T))$. Projective generators of the category $\mathfrak{B}_{d}$ are tensor products of the divided powers, while the injective cogenerators are symmetric powers. Let us recall that the $d$ th divided power functor $\Gamma^{d} \in \mathfrak{F}(A)$ and $d$-th symmetric functors $S^{n}$ are defined by

$$
\Gamma^{d}(M)=\left(M^{\otimes d}\right)^{\Sigma_{d}}, \quad S^{n}(M)=\left(M^{\otimes d}\right)_{\Sigma_{d}} .
$$

Here tensor products are taken over $A, \Sigma_{d}$ is the symmetric group on $d$ letters, which acts on the $d$-th tensor power by permuting of factors, $M \in \mathbf{F}(A)$ and $X^{G}$ (resp. $X_{G}$ ) denotes the module of invariants (resp. coinvariants) of a $G$-module $X$, where $G$ is a group.

For a functor $T \in \mathfrak{F}(A)$ we let $\widetilde{T} \in \mathfrak{F}\left(\mathbb{F}_{p}\right)$ be the functor defined by

$$
\widetilde{T}(V)=T(V \otimes A) .
$$

According to Pirashvili-Waldhausen [13, Theorem 4.1] the groups $\mathrm{HML}_{i}\left(\mathbb{F}_{p}, \widetilde{T}\right)$ have an $A-A$-bimodule structure. The left action comes from the fact that $T$ has values in the category of left $A$-modules, while the right action comes from the fact that $T$ is defined on $\mathbf{F}(A)$. In particular it uses the action of $T$ on the maps $l_{a}: X \rightarrow X$, where $a \in A, X \in \mathbf{F}(A)$ and $l_{a}$ is the multiplication on $a$. Since $T\left(l_{a}\right)=l_{a^{d}}$ if $T$ is a strict homogeneous polynomial functor of degree $d$ Friedlander-Suslin [7], the bimodule $\mathrm{HML}_{i}\left(\mathbb{F}_{p}, \tilde{T}\right)$ is of the form $\Phi^{n}(M)$ provided $d=p^{n}$.

Theorem 9 Let $d>1$ be an integer and let $A$ be a commutative $\mathbb{F}_{p}$-algebra. Then $\mathrm{HML}_{\bullet}\left(A, \Gamma^{d}\right)=0$ if $d$ is not a power of $p$. If $d=p^{n}$ and $n>0$, then

$$
\mathrm{HML}_{i}\left(A, \Gamma^{d}\right)=0 \text { if } i \neq 2 p^{n} t, t \geq 0
$$

and

$$
\mathrm{HML}_{i}\left(A, \Gamma^{d}\right)=\psi^{n}(A) \text { if } i=2 p^{n} t, t \geq 0 .
$$

In particular HML. $\left(A, \Gamma^{d}\right)=0$ provided $A$ is an algebra over a field $K$ of characteristic $p>0$ with $\operatorname{Card}(K)>d$. 
Proof According to Pirashvili-Waldhausen [13, Theorem 4.1] and Pirashvili [12] there exists a functorial spectral sequence:

$$
E_{p q}^{2}=\mathrm{H}_{p}\left(A, \mathrm{HML}_{q}\left(\mathbb{F}_{p}, \tilde{T}\right)\right) \Longrightarrow \mathrm{HML}_{p+q}(A, T) .
$$

For $T=\Gamma_{A}^{n}$ one has $\widetilde{T}=\Gamma_{\mathbb{F}_{p}}^{n} \otimes A$. Here we used the notation $\Gamma_{A}^{n}$ in order to emphasize the dependence on the ring $A$. By the result of Franjou, Lannes and Schwartz [6], $\mathrm{HML}_{i}\left(\mathbb{F}_{p}, \tilde{T}\right)$ vanishes unless $d=p^{n}$ and $i=2 p^{n} t, t \geq 0$. Moreover in these exceptional cases $\mathrm{HML}_{i}\left(\mathbb{F}_{p}, \widetilde{T}\right)$ equals to $\Phi^{n}(A)$ (as an $A-A$-module). Hence the spectral sequence together with Theorem 5 gives the result.

Corollary 10 Let $A$ be a commutative algebra over a field $K$ of characteristic $p>0$ with $\operatorname{Card}(K)>d>1$. If $T$ is a strong homogeneous polynomial functor of degree $d$. Then

$$
\mathrm{HML} \bullet(A, T)=0=\mathrm{HML}^{\bullet}(A, T) \text {. }
$$

Proof We already proved that the result is true if $T$ is a divided power. By the well-known vanishing result (Pirashvili [11]) the result is also true if $T=T_{1} \otimes T_{2}$ with $T_{1}(0)=0=T_{2}(0)$. Since any object of $\mathfrak{P}_{d}$ has a finite resolution which consists with finite direct sums of tensor products of divided powers [7] the result follows.

\section{References}

[1] M Bökstedt, The topological Hochschild homology of $\mathbb{Z}$ and $\mathbb{Z} / p$ unpublished manuscript

[2] L Breen, Extensions du groupe additif, Inst. Hautes Études Sci. Publ. Math. 48 (1978) 39-125 MR516914

[3] L L Èsakia, Heyting algebras I: Duality theory (Russian), Metsniereba, Tbilisi (1985) MR847050

[4] V Franjou, E M Friedlander, T Pirashvili, L Schwartz, Rational representations, the Steenrod algebra and functor homology, Panoramas et Synthèses 16, Société Mathématique de France, Paris (2003) MR2117525

[5] V Franjou, E M Friedlander, A Scorichenko, A Suslin, General linear and functor cohomology over finite fields, Ann. of Math. (2) 150 (1999) 663-728 MR1726705

[6] V Franjou, J Lannes, L Schwartz, Autour de la cohomologie de Mac Lane des corps finis, Invent. Math. 115 (1994) 513-538 MR1262942

[7] E M Friedlander, A Suslin, Cohomology of finite group schemes over a field, Invent. Math. 127 (1997) 209-270 MR1427618 
[8] M Jibladze, T Pirashvili, Cohomology of algebraic theories, J. Algebra 137 (1991) 253-296 MR1094244

[9] J-L Loday, Cyclic homology, second edition, Grundlehren der Mathematischen Wissenschaften 301, Springer, Berlin (1998) MR1600246

[10] S Mac Lane, Homology, Grundlehren der Mathematischen Wissenschaften 114, Springer, Berlin (1963) MR0156879

[11] T I Pirashvili, Higher additivizations, Trudy Tbiliss. Mat. Inst. Razmadze Akad. Nauk Gruzin. SSR 91 (1988) 44-54 MR1029006

[12] T Pirashvili, Spectral sequence for Mac Lane homology, J. Algebra 170 (1994) 422428 MR1302848

[13] T Pirashvili, F Waldhausen, Mac Lane homology and topological Hochschild homology, J. Pure Appl. Algebra 82 (1992) 81-98 MR1181095

[14] D Quillen, On the (co-) homology of commutative rings, from: "Applications of Categorical Algebra (Proc. Sympos. Pure Math., Vol. XVII, New York, 1968)”, Amer. Math. Soc., Providence, R.I. (1970) 65-87 MR0257068

[15] S Schwede, Stable homotopy of algebraic theories, Topology 40 (2001) 1-41 MR1791267

Department of Mathematics, University of Leicester

Leicester, LE1 7RH, UK

tp59@le.ac.uk

Received: 14 March 2007 